\title{
Stratégies énergie domestique et gestion durable des ressources forestières au Niger et au Mali : gestion, domanialité, fiscalité et contrôle forestier
}

Alain BERTRAND ${ }^{1}$

Pierre MONTAGNE ${ }^{2}$

1 Edenia Consult Tanja

28, rue Assad Ibn Al Forat Marshan

Tanger

Maroc

2 Cirad

Département Environnements

et sociétés

Unité de recherche en partenariat

Forêt et biodiversité

BP 853

Antananarivo

Madagascar

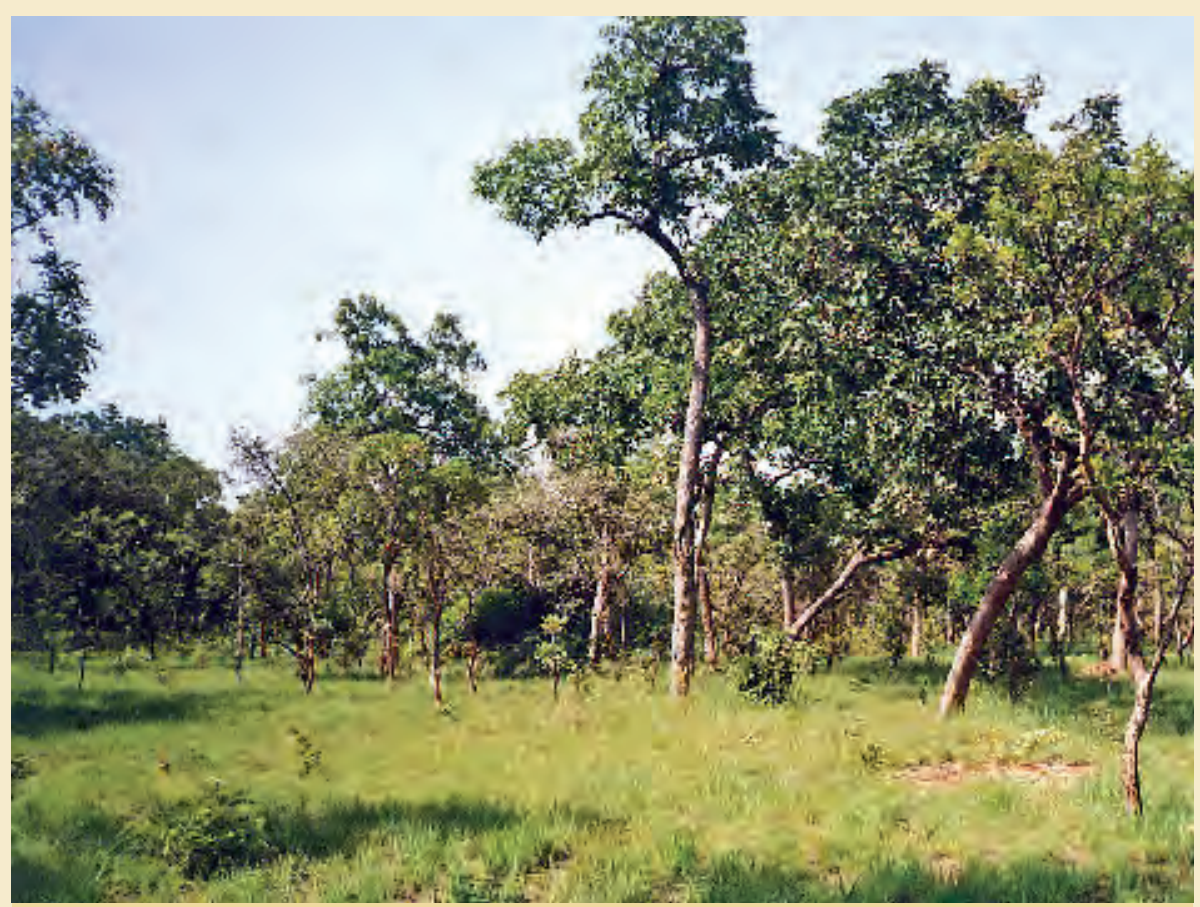

Savane boisée à Isoberlinia doka au sud du Mali, en 2007. Photo A. Bertrand. 


\section{RÉSUMÉ}

\section{STRATÉGIES ÉNERGIE DOMESTIQUE ET GESTION DURABLE DES RESSOURCES FORESTIÈRES \\ AU NIGER ET AU MALI : GESTION, DOMANIALITÉ, FISCALITÉ ET CONTRÔLE FORESTIER}

Les stratégies d'énergie domestique ont été mises en œuvre à grande échelle au Niger puis au Mali à partir des années 1990. Avec plus d'une décennie de recul, il est possible de dresser un bilan comparatif de la mise en œuvre de ces stratégies au Niger et au Mali. Ce bilan fait apparaître, audelà des similitudes, des différences fondamentales. L'article se focalise sur les résultats en termes d'aménagement forestier et de gestion durable des ressources ligneuses. Si le bilan est largement positif au Niger, il n'en est pas de même au Mali. Les modalités de la fiscalité forestière et du contrôle forestier expliquent ces différences. Le Niger et le Mali sont donc maintenant confrontés à leurs propres nouveaux défis qui s'avèrent différents. Le Niger doit assurer la généralisation de la gestion forestière par les marchés ruraux. Quant au Mali, il doit reprendre à la base la réforme de la réglementation et de la fiscalité forestières dans le cadre de la décentralisation.

Mots-clés : stratégie énergie domestique, aménagement, fiscalité, contrôle, Mali, Niger.

\section{ABSTRACT}

\section{DOMESTIC ENERGY STRATEGIES AND SUSTAINABLE MANAGEMENT OF FOREST RESOURCES IN NIGER AND MALI: MANAGEMENT, PUBLIC PROPERTY REGIME, FOREST TAXATION AND FORESTRY ASSESSMENT}

Domestic energy strategies were implemented on a large scale in Niger and subsequently in Mali as from the 1990s. With more than a decade of hindsight, a comparative review can now be made of the implementation of these strategies in the two countries. The review has highlighted fundamental differences underlying apparent similarities. This article focuses on results in terms of forest planning and sustainable management of wood resources. While the outcome is broadly positive in Niger, this is not the case for Mali. The reasons for this difference lie in the different systems of forest taxation and law enforcement. The new challenges that now face Niger and Mali are therefore different. While the challenge for Niger is to introduce forest management across the country through its rural markets, Mali needs to review the foundations of its reform of forest regulations and taxation in the context of decentralization.

Keywords: domestic energy strategies, planning, taxation, enforcement, Mali, Niger.

\section{RESUMEN}

\author{
ESTRATEGIAS DE ENERGÍA \\ DOMÉSTICA Y MANEJO SOSTENIBLE \\ DE RECURSOS FORESTALES \\ EN NÍGER Y MALÍ: ORDENACIÓN, \\ REGIMEN DE PROPRIEDAD, \\ FISCALIDAD Y CONTROL FORESTAL
}

Las estrategias de energía doméstica se implementaron a gran escala en Níger y, más tarde, en Malí a partir de los años noventa. Con más de una década de perspectiva, es posible establecer un balance comparativo de la aplicación de estas estrategias en ambos países. Este balance, además de aspectos similares, refleja diferencias fundamentales. El artículo se centra en los resultados en términos de ordenación forestal y manejo sostenible de los recursos leñosos. Si el balance es muy positivo en Níger, no ocurre lo mismo con Malí. Las diferencias se explican por los modos de fiscalidad y control forestales. Níger y Malí se hallan, pues, frente a nuevos retos, que difieren entre sí. Níger debe garantizar la generalización del manejo forestal por los mercados rurales. Malí, por su parte, debe reorganizar desde la base la reforma de la normativa y la fiscalidad forestal en el marco de la descentralización.

Palabras clave: estrategias de energía doméstica, ordenación, fiscalidad, control, Malí, Níger 


\section{Introduction}

Les décennies 1980 et 1990 , années du changement en Afrique de l'Ouest, ont vu la mise en place de structures institutionnelles nouvelles où la place de la société civile a été de plus en plus importante. Chaque pays a vécu cette transition à son rythme, selon son histoire et sa tradition. Ces décennies ont aussi été dans ces pays celles des politiques de décentralisation avec différents niveaux de collectivités territoriales.

Cette évolution a permis des changements profonds des modes de gestion des ressources naturelles. Au Niger depuis 1990, au Mali depuis 1993, les réflexions ont conduit à revoir les modes de gestion à partir de nouveaux schémas institutionnels et organisationnels (figure 1), délaissant la gestion centralisée des ressources par l'État à travers l'administration chargée des forêts. L'année de la grande sécheresse, 1984, a été la charnière : le Niger a fait alors le choix de l'aménagement forestier, qui s'est traduit politiquement en 1989 par ce qui a été dénommé la «stratégie énergie domestique » (Sed). Cette stratégie a été mise en œuvre dans le cadre du projet Énergie II de la Banque mondiale ${ }^{1}$ en appui au service forestier nigérien (figure 2). La réalisation des schémas directeurs d'approvisionnement de Niamey, puis de Maradi et de Zinder, a permis d'organiser et de planifier l'approvisionnement en bois énergie des villes. Cette planification de la gestion des formations forestières a été développée en 1990 au Niger et en 1997 au Mali. Son objectif est de localiser les ressources dans le bassin d'approvisionnement de la ville, d'identifier les flux, les acteurs, la provenance du bois, les chaînes (transporteurs, charbonniers, bûcherons, commerçants). À l'échelle des massifs

\footnotetext{
1 Financé par le Danemark, dans lequel le Cirad est intervenu, avec les partenaires des bureaux d'études Seed (Stratégie, Énergie, Environnement, Développement) et Marge (Marchéage et gestion de l'environnement).
}

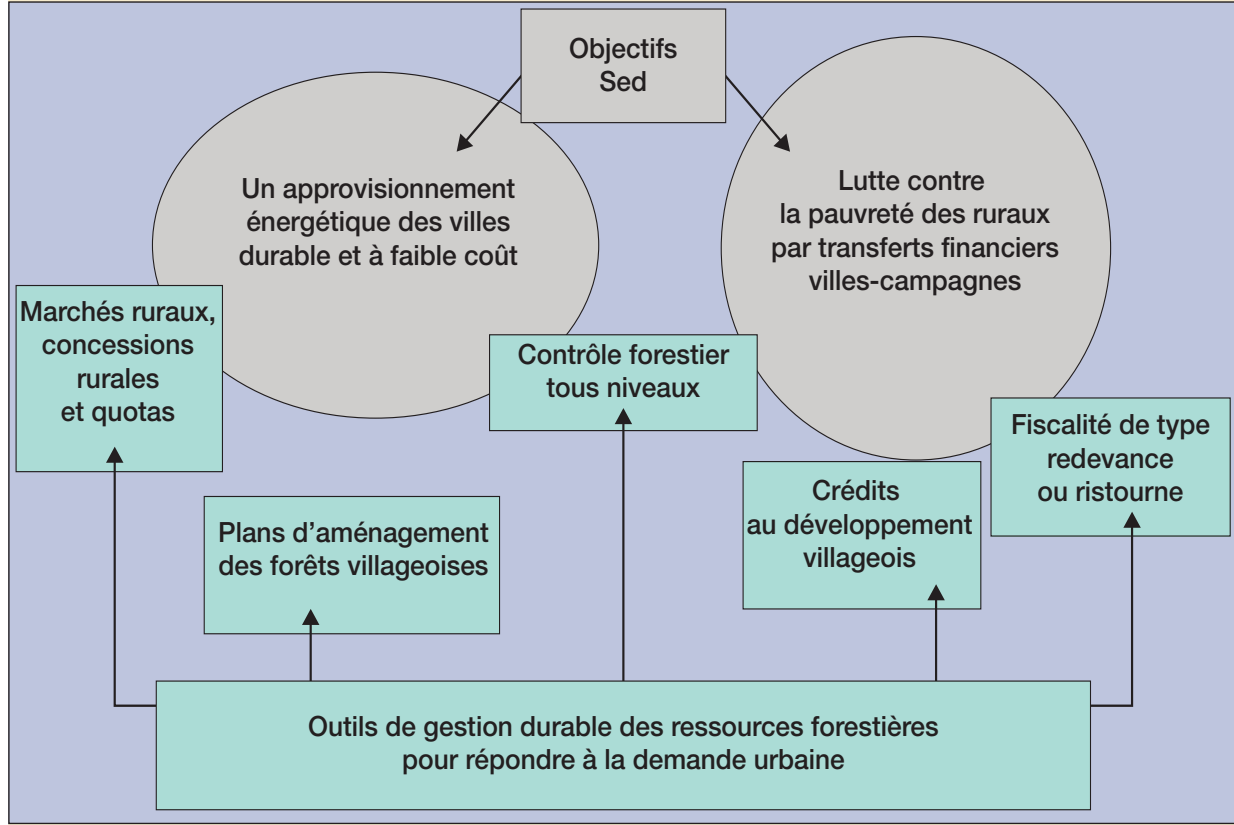

Figure 1.

Les objectifs et les instruments des stratégies énergie domestique. forestiers, cet exercice définit les actions de transfert de la gestion des ressources aux communautés villageoises et la création de marchés ruraux du bois énergie. L'État demande alors aux paysans-bûcherons de gérer leurs propres ressources dans un cadre légal nouveau (fixé au Niger par une calité incitative spécifique, permet la mise en place d'un suivi et d'un contrôle décentralisés et autofinancés.

Àce jour et avec cette ampleur, les expériences développées au Niger et au Mali pour assurer le transfert de gestion des forêts pour la production de bois énergie de l'État aux populations restent uniques en Afrique. Leurs caractéristiques communes et principales sont, d'une part, d'avoir mis en place un cadre légal, législatif et réglementaire, qui garantit aux populations riveordonnance de 1992) qui, par une fis- raines les droits de commercialisation exclusifs de leurs ressources et, d'autre part, de vouloir développer le dispositif villageois de production à l'échelle des bassins d'approvisionnement des villes pour réduire la part des exploitations souvent illicites de commerçants-transporteurs et d'instaurer une fiscalité forestière différentielle selon les modes d'exploitation de la ressource. Les deux pays diffèrent par le pas de temps de leur processus de décentralisation de l'État, mais aussi par des modalités très variées de la fiscalité différentielle et du contrôle forestier.

Ainsi, dans ces deux pays, une réforme réglementaire précède et accompagne le transfert de gestion des ressources de l'État aux populations riveraines des massifs forestiers, à des étapes différentes de la décentralisation.

\section{Nota bene}

Bois et forêts des tropiques a de longue date consacré, au cours de la décennie 1990, de multiples articles aux stratégies énergie domestique au Sahel et aux marchés ruraux du bois énergie. Les lecteurs qui n'auraient pas lu ces précédents articles pourront s'y reporter puisqu'ils sont accessibles sur le site web de Bois et forêts des tropiques (wwww.cirad-bft.fr). 


\section{Les stratégies énergie domestique au Niger et au Mali}

La stratégie énergie domestique au Niger a combiné de façon cohérente une nouvelle politique énergétique et une nouvelle politique forestière. Proposée en 1986 (MAdon, MatLy, 1986), elle fut mise en application à partir de 1989. Les constats de départ de la stratégie énergie domestique étaient les suivants :

- le bois énergie était (et reste) le combustible domestique de base et un produit de première nécessité pour des populations urbaines en forte croissance ; en conséquence, le consommateur est confronté à une demande socialement incompressible ;
- les dynamiques d'exploitation prédatrices des acteurs économiques dégradaient la périphérie des grandes villes ; les études concluaient à la nécessité de gérer globalement et rationnellement des ressources jugées suffisantes et à celle d'orienter spatialement les dynamiques d'exploitation du bois énergie vers des zones moins fragiles;

- les filières d'approvisionnement en bois énergie représentaient un chiffre d'affaires global annuel de plus de dix milliards de francs Cfa (monnaie répandue dans les pays d'Afrique de l'Ouest), de l'ordre d'un milliard et demi de francs Cfa pour la seule ville de Niamey. Un secteur d'importance économique majeure était délaissé et ignoré de la puissance publique ;

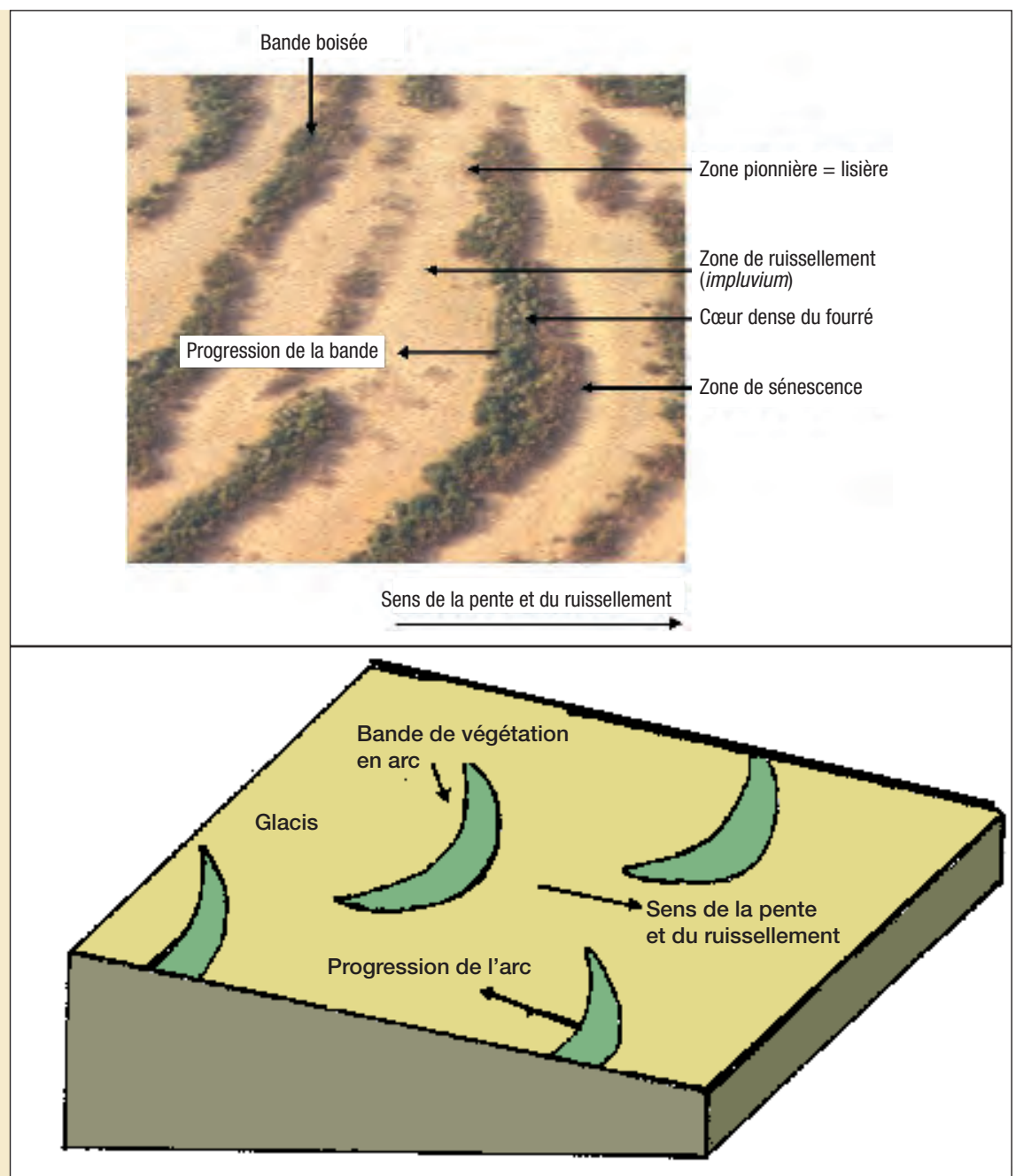

Figure 2.

Illustrations du fonctionnement hydrique des formations contractées.
- les dynamiques d'exploitation prédatrices des commerçants-transporteurs excluaient les populations rurales des revenus de l'exploitation du bois énergie et les démotivaient de gérer durablement les ressources de leur terroir.

La stratégie énergie domestique visait à répondre simultanément à des objectifs différents :

- assurer durablement, au moindre coût économique et social, l'approvisionnement des consommateurs urbains en bois énergie ;

- mettre en place des modes de gestion durables des ressources naturelles exploitées pour approvisionner en bois énergie les villes.

La mise en place des forêts villageoises et des marchés ruraux de bois énergie s'est d'abord fondée, d'une part, sur la reconnaissance des communautés locales comme interlocuteurs légitimes de l'administration et, d'autre part sur l'élaboration d'un contrat de transfert de gestion des ressources locales de production de bois énergie, sur la reconnaissance des droits exclusifs de chaque communauté sur les ressources de son terroir et enfin sur la mise en place préalable d'un cadre législatif et fiscal incitatif pour les communautés et les acteurs économiques. Ainsi, les forêts villageoises et les marchés ruraux de bois énergie au Niger ont permis de réaliser la réaffectation légale d'un espace forestier considéré comme légitime par les communautés villageoises. S'est ainsi (re)mis en place un maillage du territoire permettant une gestion locale par les populations sur la base d'un monopole local de gestion, d'exploitation et de valorisation reconnu à ces populations à travers un marché rural de bois énergie.

Chaque forêt villageoise est délimitée et permanente. Il était prévu l'immatriculation foncière des forêts villageoises. Un quota annuel d'exploitation et un plan d'aménagement constituent les bases techniques garantissant à l'administration forestière que la gestion forestière sera durable et que l'exploitation du bois énergie sera réalisée selon les règles techniques validées (figure 2). 


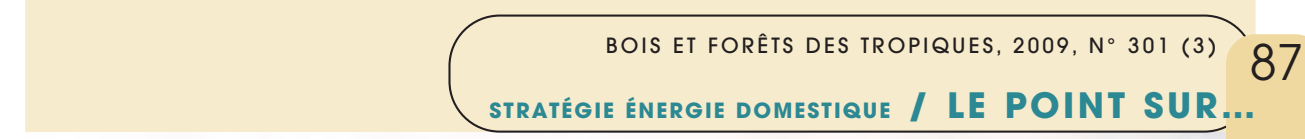

Compte tenu de l'importance économique de la filière bois énergie et de la multiplicité des acteurs, la gestion publique ne peut être qu'indirecte et basée sur des incitations économiques, plus que sur des interdictions ou des réglementations. C'est ce qui justifie la mise en place d'une fiscalité forestière différentielle et d'un quota annuel d'exploitation au sein de chaque marché rural. Ces outils économiques et réglementaires combinés ont pour fonction d'encadrer les acteurs de la filière et de les orienter vers des pratiques compatibles avec la gestion durable des ressources forestières du Niger.

Pour chaque marché rural, une structure locale de gestion, avec le monopole local de vente du bois énergie, doit assurer la commercialisation du bois énergie exploité par des bûcherons villageois groupés au sein d'une association des bûcherons.

La fiscalité forestière du bois énergie au Niger a été redéfinie dans le cadre de la stratégie énergie domestique avec :

- une fiscalité différentielle décroissante entre l'exploitation incontrôlée (taxée au niveau 3) et l'exploitation par les marchés ruraux (classés en deux types, à savoir les marchés ruraux orientés taxés au niveau 1,5 et les marchés ruraux contrôlés taxés au niveau 1) (tableau I) ; - au sein des marchés ruraux, un dispositif de collecte de la taxe par le marché rural qui permet une meilleure

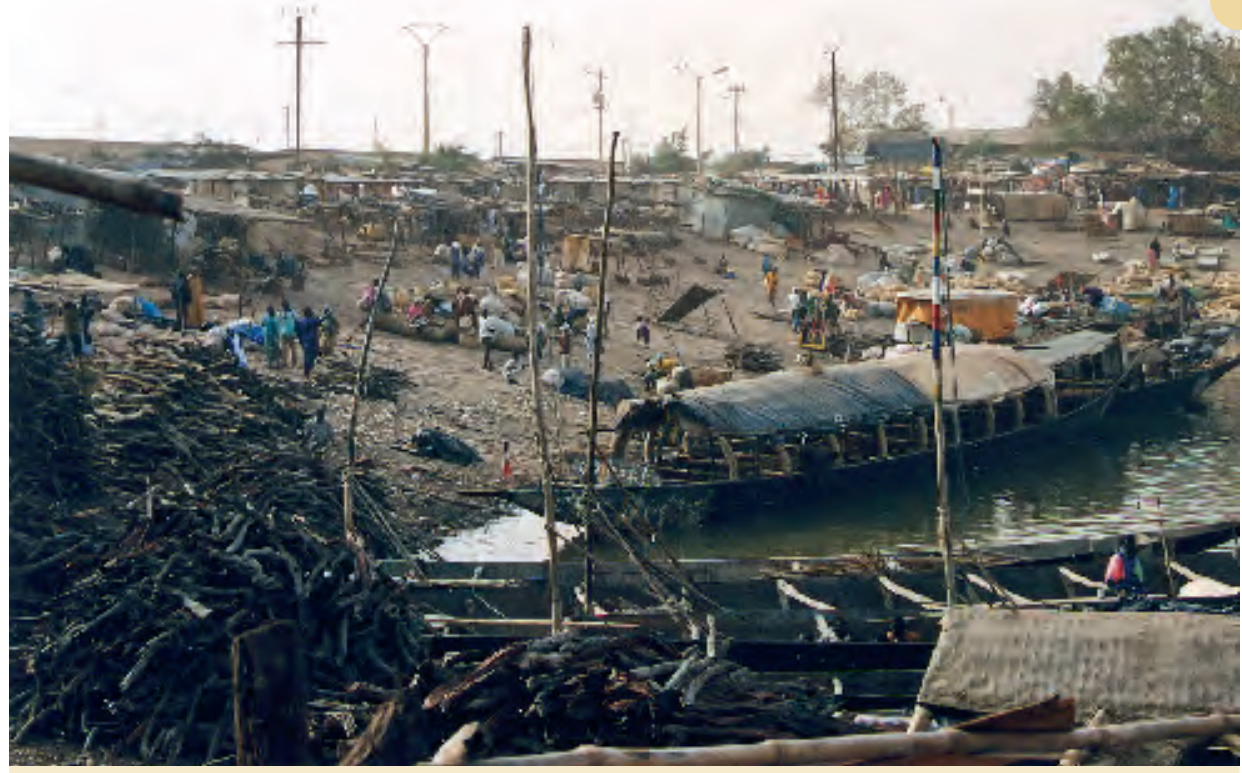

Le port à bois de Mopti, au Mali, en 1999.

Photo A. Bertrand.

visibilité de la taxe et une valorisation optimale de la ressource; il y a enfin remise de coupons justifiant du paiement de la taxe ;

- pour l'exploitation incontrôlée, acquittement de la taxe auprès des services déconcentrés de l'environnement et vérification du paiement de la taxe par le contrôle forestier permanent (vingt-quatre heures sur vingtquatre) à l'entrée en ville ;

- une répartition du produit de la taxe forestière prélevée au niveau du marché rural entre le budget de la communauté rurale, des travaux forestiers (appui aux régénérations naturelles), le budget de l'arrondissement (la commune), le fonds du contrôle forestier, le budget de l'État ;
- une autre répartition de la taxe forestière sur l'exploitation incontrôlée (entrée en ville) entre le fonds du contrôle forestier et le budget de l'État.

Pour être viable, l'aménagement forestier en gestion villageoise doit être aussi simple que possible et basé sur des règles compréhensibles et applicables par tous. En conséquence, le coût de l'aménagement des forêts villageoises au Niger est de moins de cinq dollars US par hectare. Il peut être pris en charge dans le cadre des revenus locaux de la fiscalité différentielle sur le bois énergie.

Quant au Mali, la stratégie énergie domestique fut établie entre 1993 et 1996 sur les mêmes bases mais selon des modalités d'application différentes qui n’ont pas été sans conséquences sur la situation actuelle.

Tableau I.

Décentralisation et transferts de gestion au Niger et au Mali.

Décentralisation et création des com
$\begin{aligned} & \text { Transfert de gestion de forêts aux } \\ & \text { communautés rurales. }\end{aligned}$
communautés rurales.

$$
\text { Niger }
$$

Depuis 2004, un seul scrutin communal en 2004.

Depuis 1992 : ordonnance 92-037 confirmée par la loi forestière 2004-040. Près de 300 transferts de gestion réalisés avec de bons résultats pour la durabilité forestière.

Expériences de fiscalité forestière différentielle.
Expérience au niveau national depuis 1992, assez concluante malgré un contrôle forestier insuffisant ; concerne le bois de feu.
Mali

Depuis 1993, plusieurs élections communales successives.

Depuis 1997, lois de 1997. Près de 400 transferts de gestion réalisés avec des résultats insuffisants pour la durabilité forestière.

Expérience au niveau national depuis 1997 non concluante (contrôle forestier non efficace), concernant le bois énergie. 


\section{L'approche} comparative des stratégies énergie domestique au Niger et au Mali

Plus de dix ans après ces bouleversements, des dispositifs législatifs et réglementaires novateurs, de nouvelles politiques forestières ont été promulgués et commencent à produire leurs effets. Il est intéressant de constater que ces changements se sont effectués de façon simultanée et parallèle mais qu'ils aboutissent à des résultats qui, tout en étant différents voire opposés, sont similaires dans leur trame (tableau II).
Ces transferts de la gestion locale des forêts réalisés à grande échelle contrastent avec la multiplicité des opérations pilotes ou de rechercheaction menées dans le même temps dans de très nombreux autres pays (sauf Madagascar qui est à un niveau comparable) (tableau III). Seule l'Inde, avec la mise en place des actions de " joint-forestry », a réalisé des actions de transfert de gestion d'ampleur comparable (Roy, 1995). Cela différencie les expériences de transfert de gestion en Afrique francophone de celles mises en œuvre dans les pays anglophones d'Afrique où le processus de réforme réglementaire a commencé pratiquement avec une décennie de retard, à la fin des années 1990 (Alden Wily, 2000). L'approche comparative entre Mali et Niger permet d'identifier des facteurs clés de la gestion durable des ressources forestières et de la gouvernance locale contrac-

Tableau II.

Les impacts des transferts de gestion sur le développement et la gestion locale forestière au Niger et au Mali.

Niger

Réduction de l'exode rural saisonnier ou à long terme, accroissement des revenus des populations rurales.

\section{Mali}

Accroissement des revenus des populations rurales et contribution effective du bois énergie aux revenus des populations et au développement local.

Pas d'informations sur ce sujet.

Réduction sensible du nombre et de l'intensité des conflits.

Très bon respect des règles d'exploitation, des textes forestiers et de la fiscalité forestière locale.

Mauvais respect (environ $30 \%$ ) des règles d'exploitation et des textes forestiers, comme de la fiscalité forestière locale.

Respect quasi général du quota.

Respect du quota annuel d'exploitation.

Évaluation par les villageois de l'évolution de l'état de la forêt villageoise.

Surveillance de la forêt villageoise par les villageois.

Évaluation de la capacité locale de gestion de l'association locale de gestion de la forêt villageoise et de commercialisation des produits forestiers.

Structuration et fonctionnement de l'association locale de gestion de la forêt villageoise et de commercialisation des produits forestiers.
$75 \%$ des habitants observent un accroissement du couvert de la forêt villageoise.

Effective dans $70 \%$ des cas.

Bonne gestion en général et bon respect des procédures administratives malgré des dérapages isolés.

Tous les marchés ruraux ont une structure locale de gestion normalement constituée et fonctionnelle.
Non de façon quasi générale, quota non respecté.

$60 \%$ à $100 \%$ des habitants observent une réduction du couvert de la forêt villageoise.

Effective dans $60 \%$ des cas.

Cas de mauvaise gestion et de besoins de formation mais en général nonrespect des procédures administratives.

De $30 \%$ à $50 \%$ des marchés ruraux n'ont pas une structure rurale de gestion normalement constituée et fonctionnelle. 
tuelle dans un contexte de décentralisation (Bertrand, Montagne, 2008). Les deux pays ont en commun, malgré des rythmes et des politiques de décentralisation spécifiques, de définir la commune comme la plus petite collectivité territoriale décentralisée. Le nombre total des transferts de gestion des forêts aux populations rurales dépasse plusieurs centaines et leur durée moyenne autorise l'établissement d'un premier bilan.
Les populations ont recouvré une forte légitimité perdue de leur gestion coutumière locale sur des espaces communs et cela semble dans certains cas leur suffire pour émettre un avis positif. Par contre, d'autres conditions semblent déterminer le respect ou le non-respect des dispositions institutionnelles, réglementaires ou fiscales mises en place et donc la gestion conservatoire ou la mise en coupe réglée des forêts villageoises.
L'impact positif sur le développement local induit, même si elle est lente, une évolution de la reconnaissance sociale des bûcherons et charbonniers (tableau IV). Ces derniers passent du statut peu enviable de "clandestin » sous-traitant exploité par les donneurs d'ordre à celui d'acteur à qui est confiée la responsabilité d'améliorer les conditions de production (meilleures techniques de coupe, de carbonisation, respect des quotas et des rotations, etc.).

Tableau III.

Les impacts sociaux et environnementaux des transferts de gestion au Niger et au Mali.

Niger

Amélioration de l'image sociale et du statut des bûcherons.

Impacts sociaux de la forêt villageoise et de la création des marchés ruraux de bois énergie.

Évaluation de la bonne gouvernance des structures locales de gestion des forêts villageoises et des marchés ruraux.

Impacts environnementaux et forestiers et respect des limites des forêts villageoises.
Faible transparence de la gestion des structures locales de gestion, néanmoins elles sont fonctionnelles.

Réduction des défrichements agricoles en forêt (limités aux réserves foncières prévues à cet effet).

Augmentation sur 15 ans des volumes moyens de bois vert sur pied (60 sites d'observation).

Gestion locale durable des forêts villageoises effective.
Mali

Non évalué

Non évalué

Non évalué

Tableau IV.

Conséquences comparées des stratégies énergie domestique au Niger et au Mali : des différences fondamentales.

Niger

Contrôle forestier local par les Slg efficace.

Contrôle forestier centralisé de l'administration globalement inefficace.

Les marchés ruraux sont globalement pérennes.

Respect des aménagements forestiers villageois et des limites des forêts villageoises, diminution des défrichements dans les forêts villageoises.

Perception efficace à la source de taxes forestières et revenus de la fiscalité forestière conséquents pour les communautés rurales et pour les communes où sont installés des marchés ruraux.

L'exploitation du bois énergie devient une activité villageoise rémunératrice et considérée socialement ; impacts sur le développement rural local.
Mali

Contrôle forestier inefficace fonctionnant au profit des commerçants-transporteurs et des revenus " parallèles » des agents forestiers.

Multiplication des points de vente incontrôlés de bois énergie et dépérissement des marchés ruraux de bois.

Disparition de fait des aménagements forestiers, remplacés par une exploitation complètement incontrôlée ; les dynamiques de défrichement des forêts se poursuivent et s'amplifient.

Aucun revenu fiscal sur la fiscalité forestière pour les communautés rurales ni pour les communes.

L'exploitation du bois énergie devient une activité villageoise rémunératrice et considérée socialement ; impacts sur le développement rural local. 


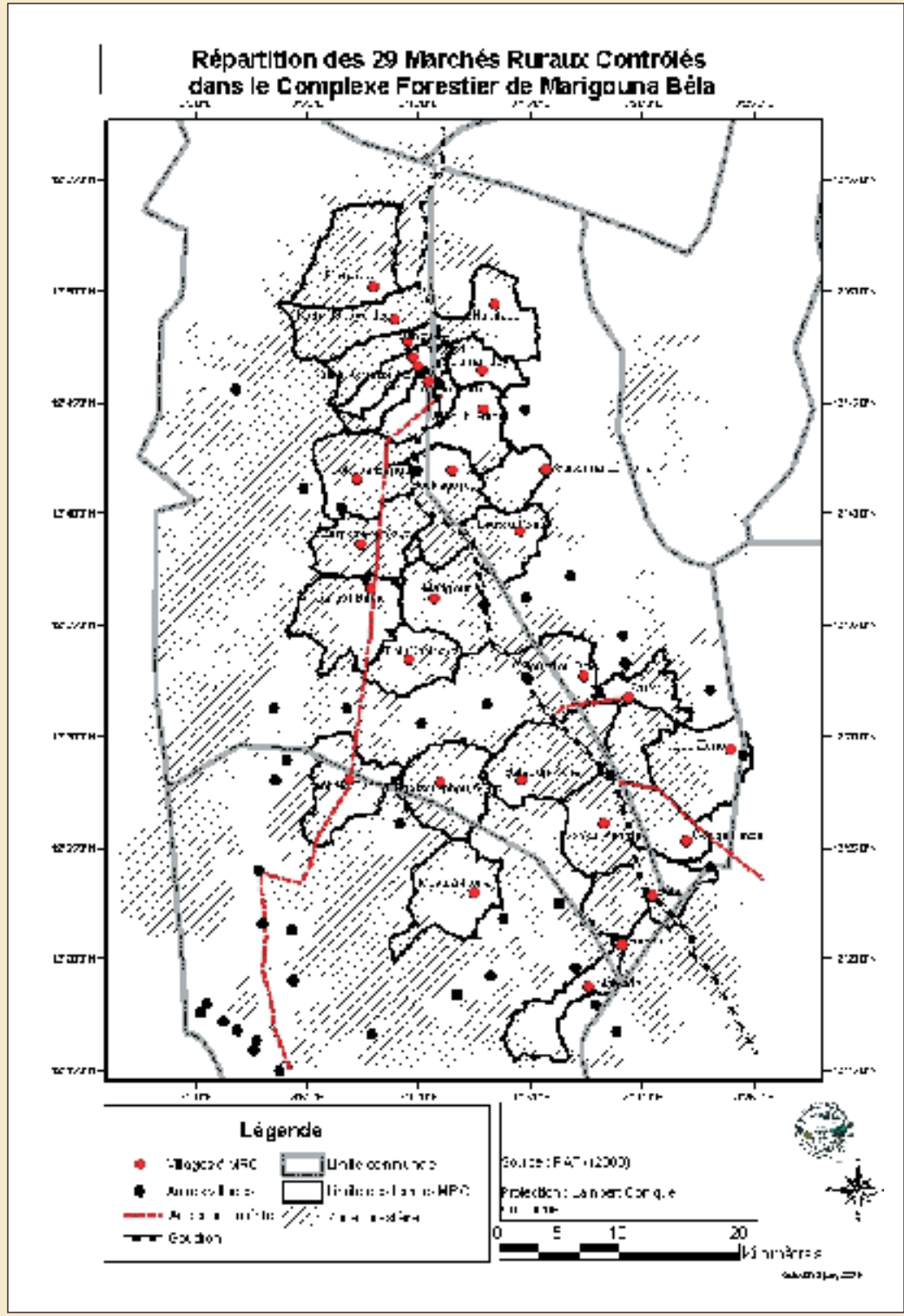

Figure 3.

Répartition des vingt-neuf marchés ruraux contrôlés dans dans le complexe forestier de Marigouna Béla.

Les communautés sont souvent considérées comme le niveau socialement légitime et efficace de mise en œuvre d'actions de gestion forestière locale. Mais leur légalité n'est pas toujours clairement définie et encore moins opérationnelle. À l'inverse, les communes, niveau de base des collectivités territoriales décentralisées, bénéficient d'une assise légale formelle. Mais elles couvrent un espace plus vaste et correspondent à une réalité sociale plus leur pérennité à long terme et de leurs modes de gestion prennent une importance stratégique non seulement au niveau local, mais aussi au niveau national. Le renforcement des droits (et donc de la légitimité et de la capacité de contrôle) des acteurs locaux sur la forêt devient une des conditions majeures et un des principaux moyens de sortie de la pauvreté pour les populations. La reconnaissance institutionnelle des niveaux communaux et communautaires comme étant ceux des interlocuteurs légitimes de l'État, à même de s'organiser pour valoriser durablement leurs ressources forestières communes, devient un enjeu majeur des politiques publiques.

La commune, entité politico-administrative, représente, par rapport à la notion souvent floue de communauté locale, un cadre potentiel pour un développement économique associé à une gestion durable de la forêt. La commune est la collectivité territoriale instituée comme ultime échelon dans les processus de décentralisation, quel que soit le pays considéré. Elle est surtout une entité politique et économique cohérente qui s'étend souvent sur une superficie importante (commune de Torodi au Niger), englobant plusieurs (voire plusieurs dizaines) villages ou communautés locales et pouvant plus ou moins couvrir certaines fonctions écologiques de la forêt (bassin versant, écosystèmes fragiles...). À une autre échelle, la notion d'intercommunalité permet de regrouper, selon des objectifs et surtout des intérêts communs, plusieurs communes (figure 3 ).

Une première caractéristique commune aux diverses expériences de réforme des politiques forestières $d u$ Niger et du Mali, auxquelles le Cirad $^{4} \mathrm{a}$ contribué, est d'être fondées sur l'affirmation par la loi d'un certain nombre de grands principes :

\footnotetext{
${ }^{2}$ Certains auteurs ont considéré, par exemple, qu'il faudrait plusieurs décennies au Mali avant que les communes acquièrent une réelle légitimité au sein des communautés villageoises de base.

${ }^{3}$ On observe parfois une course à la ressource : à l'exploitation minière incontrôlée, au défrichement, à l'appropriation foncière individuelle ou lignagère des espaces collectifs résultant directement de l'accès libre de fait institué par les réglementations coloniales reprises après les indépendances.

${ }^{4}$ Centre de coopération internationale en recherche agronomique pour le développement.
} 
- affirmer le renoncement de l'administration forestière à sa politique antérieure de répression et d'exclusion des populations rurales de la gestion forestière ;

- restaurer la légitimité de la gestion locale des ressources communes par les communautés de base ;

- ouvrir l'horizon des possibles aux yeux des populations rurales en rendant crédible et légitime la perspective d'une gestion forestière locale déléguée et contractuelle ;

- lever (ou tenter de lever) de ce fait l'opposition du légal et du légitime qui caractérisait au Niger et au Mali les relations entre l'administration forestière et les populations rurales ; - définir le cadre institutionnel de la reconnaissance juridique des communautés locales de base comme acteurs de la gestion des forêts.

\section{Des résultats spectaculaires et contrastés en matière d'aménagement forestier et de prise en compte du pastoralisme}

Au Niger, la Sed, définie en 1989 et institutionnalisée en 1992, a été mise en œuvre à travers plusieurs projets successifs ou simultanés : 1989-1998, PE II-ED (financement Danida/Banque mondiale) ; 20002002, Ped (financement Danida) ; 2001-2006, Pafn (financement Bad5) récemment achevé.
Ce dernier projet a amplifié les actions antérieures en introduisant un certain nombre d'innovations capitales.

- Le projet avait des objectifs particulièrement ambitieux avec l'élaboration de quatre plans d'aménagement des massifs forestiers de Marigouna Béla, de Baban Rafi, de la doumeraie du Goulbi N'kaba et de quelques petits massifs dans le secteur de Tahoua (Anonyme, 1996). La superficie des cinq massifs ayant finalement fait l'objet d'un plan d'aménagement représente plus de 230000 ha. Près de 141500 ha ont été placés sous exploitation et traduits en plans villageois d'aménagement forestier ou plans communaux de gestion des doumeraies (Pvaf ou Pcgd) ;

\footnotetext{
${ }^{5}$ Banque africaine de développement.
}

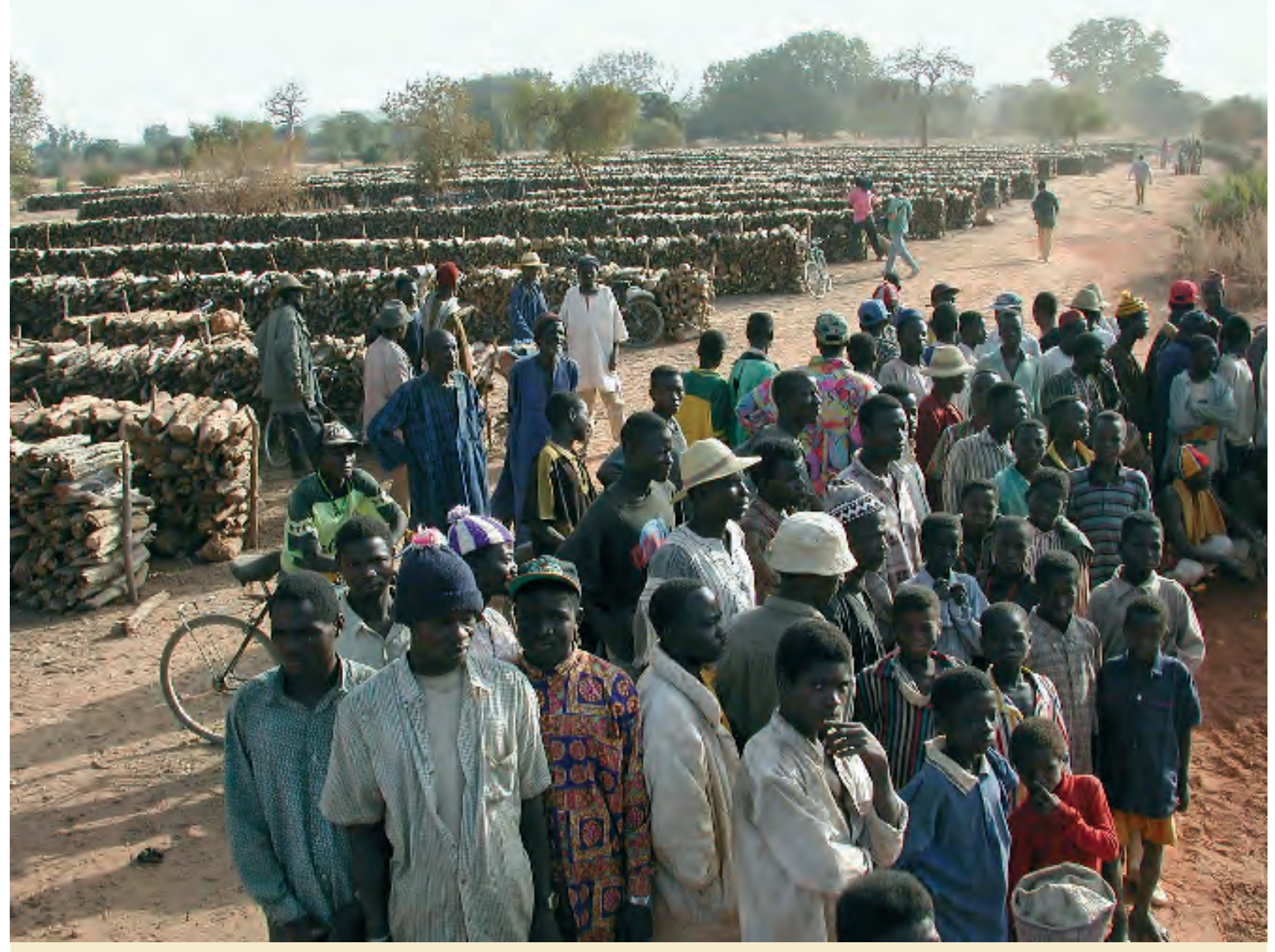

Un marché de bois de feu dans la commune de Torodi, au Niger, en 1998. Photo M. E. Loffeier. 


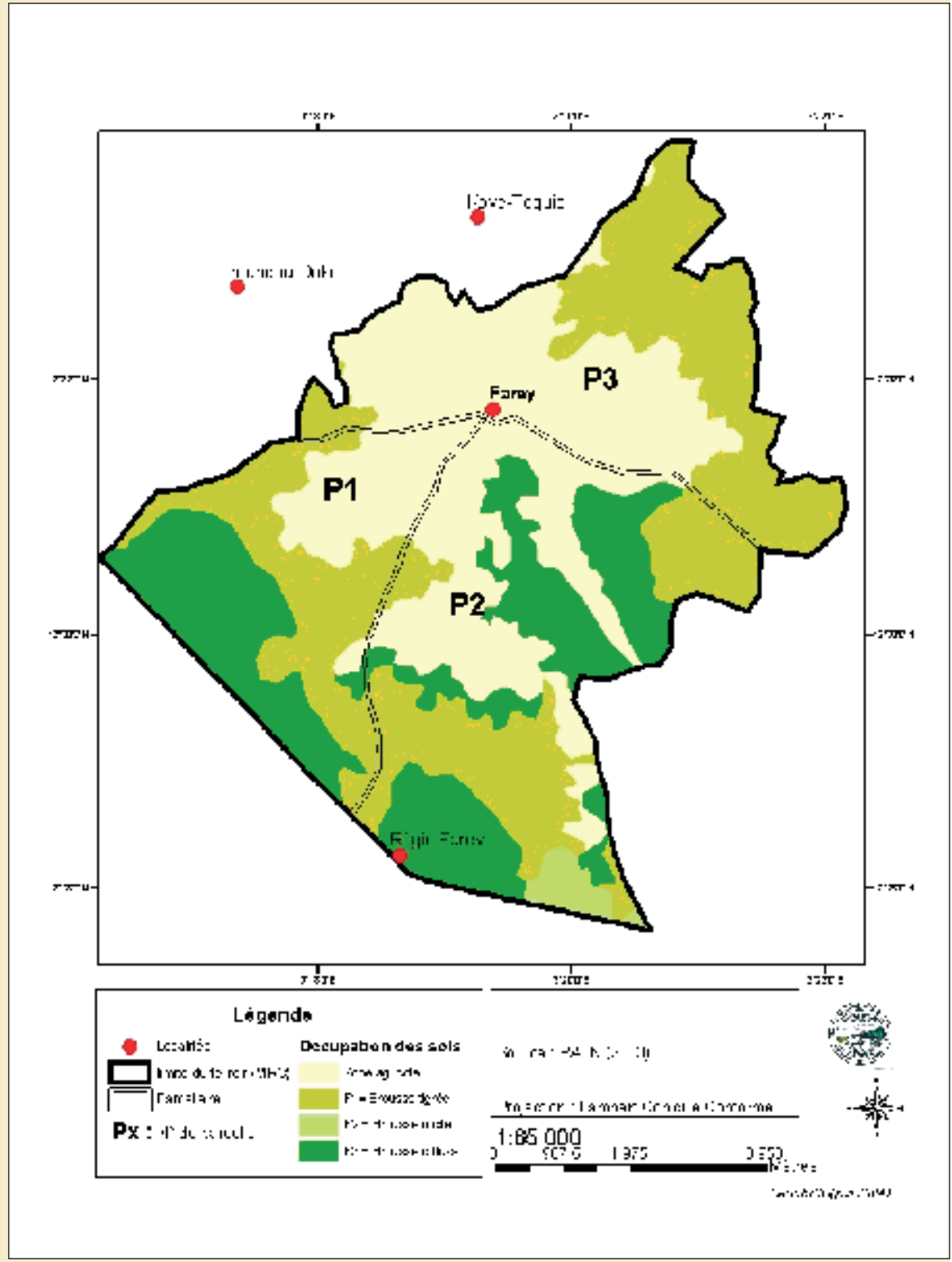

Figure 4.

Parcellaire d'exploitation du terroir forestier villageois de Farey.

7320 ha ont été inscrits en série de conservation (Baban Rafi sud et N'solo) et près de 89000 ha sont potentiellement prêts à être mis en exploitation (Marigouna Béla, Baban Rafi nord et Injinjira). Les plans d'aménagement ont ensuite été déclinés en soixante et un plans villageois d'aménagement forestier (Pvaf ; figure 4) et quatre plans communaux de gestion de la doumeraie du Goulbi N'kaba6 (Pcgd).
- L’intégration pastorale a figuré au premier plan des préoccupations tout au long du processus d'élaboration des plans d'aménagement forestier. En effet, outre la production de bois et de produits forestiers non ligneux, la forêt apporte au cheptel une contribution essentielle à ses besoins en pâturages et en eau. Or, la pression foncière cause chaque année le défrichement de superficies forestières importantes, qui est à l'origine de situations conflictuelles de plus en plus fréquentes entre agriculteurs et éleveurs et d'une marginalisation croissante de ces derniers. En conséquence, en sécurisant les systèmes de production sylvopastoraux et en prévenant les conflits ruraux, l'intégration de la dimension pastorale dans les aménagements forestiers doit contribuer à la gestion durable des ressources sylvopastorales.

Au contraire, au Mali, le fonctionnement des marchés ruraux de bois a connu et connaît de sérieuses difficultés qui peuvent être imputées au désavantage structurel des marchés ruraux par rapport à l'exploitation incontrôlée tant que le contrôle forestier est inefficace. L'exploitation incontrôlée est une activité très dynamique au Mali en général et en troisième région en particulier. Le bois, et particulièrement le bois énergie (bois de feu et/ou charbon de bois), constitue une source très appréciable de revenus complémentaires de l'agriculture pour les populations rurales. Dans ces conditions, tout le monde est à peu près rentré dans le domaine de l'incontrôlé à partir de 2004, les marchés ruraux payant les mêmes taxes que les autres, quand ils les paient. Le résultat de cette situation est l'absence de mise en œuvre de plans d'aménagement forestier ${ }^{7}$.

6 À Mayahi, le processus d'élaboration participative du plan d'aménagement de la doumeraie s'est appuyé sur les structures précommunales mises en place dans le cadre d'un projet d'appui au développement local (Padel/Pnud). 7 Sauf pour les forêts classées dans la périphérie de Bamako, qui sont de longue date le «terrain de jeu » de l'administration forestière. Cela n'y empêche pas un surpâturage structurel dû à la présence quasi permanente des troupeaux des grands éleveurs urbains « intouchables». 
Aujourd'hui, il n'existe plus, de fait, d'aménagement forestier appliqué au Mali, hormis peut-être dans les forêts classées autour de Bamako où l'administration forestière est présente. Cela ne signifie pas nécessairement que, globalement, la forêt malienne est surexploitée au-delà de sa possibilité. Cette évaluation n'a pas été faite depuis le dernier inventaire forestier réalisé avant la mise en place de la stratégie énergie domestique au Mali8.

Par contre, l'exploitation incontrôlée de fait entraîne en de nombreux lieux une surexploitation locale des ressources forestières avec des dégradations environnementales souvent irréversibles. Sans réel transfert de gestion et sans aménagement forestier villageois, la forêt malienne illustre parfaitement le théorème de G. Hardin sur la dégradation des biens sans maîtres livrés à la course à l'exploitation (HARDIN, 1968).

Les textes adoptés dans ces deux pays donnent aux populations de nouveaux droits pour la gestion locale de leurs ressources naturelles. Souvent appelées "transfert de gestion » ou «transfert de droits de gestion » ou encore « transfert de compétences de gestion », ces nouvelles règles et droits proposés aux populations riveraines sont aussi synonymes de devoirs, notamment de respect des cahiers des charges garants de la pérennité de ces ressources. Adoptées le plus souvent sur des bases contractuelles aux modalités définies par les textes, ces règles visent toutes à mettre en place de nouveaux cadres d'une gestion conservatoire de ces ressources, notamment par la mise en place de quotas (ou autres normes réglementaires ou économiques). Ces quotas varient lorsqu'il s'agit d'une exploitation - bois énergie, bois d'œuvre au sud du Mali ou tout autre produit forestier ligneux ou non ligneux ${ }^{9}$ (BERTRAND et al., 2006) qui peut être commercialisé sur des bases durables - ou d'une mise en défens totale ou partielle d'un territoire ou d'une ressource. Dans ce dernier cas, la volonté de cette mise en défens émane bien de la population concernée.
L'État devrait reconnaître l'inefficacité durable de la gestion administrative autoritaire, répressive et exclusive antérieure, et mettre fin à l'accès libre de fait résultant du nonrespect des dispositions antérieures. Il revient à l'État d'assurer un contrôle collectif local par les communautés, motivées par un transfert de gestion en bonne et due forme, restaurant par un cadre institutionnel rénové la légitimité de leur gestion coutumière.

Les communes nouvellement créées et manquant de moyens et de revenus durables pour initier et soutenir le développement local, la gestion locale et la valorisation (donc l'exploitation) des ressources naturelles apparaissent comme le moyen le plus rapide et le plus sûr de dégager des recettes et d'asseoir une fiscalité locale.

Quant aux communautés, elles devraient restaurer au plus vite la légitimité de leur gestion coutumière contestée depuis un siècle, en commençant par les ressources ou les espaces les plus « menacés » ou les plus « aliénés » par l'administration (par exemple les forêts classées). Il s'agit aussi d'assurer au plus vite leur monopole local de l'accès et de l'exploitation de ces ressources face aux intervenants extérieurs.

En période d'ajustement structurel et de réduction drastique et durable des budgets et des moyens des administrations des pays africains, les réformes des politiques forestières vers la gestion locale des ressources renouvelables forestières ne pouvaient être mises en œuvre que dans le cadre de solutions (institutionnelles, organisationnelles, techniques, etc.) à très faibles coûts récurrents. Cela implique de donner aux populations rurales concernées la responsabilité de la gestion locale des ressources, c'est-à-dire :

- de leur faire supporter une partie du coût récurrent de cette gestion ;

- de leur transférer tout ou une partie substantielle du bénéfice des fonctions essentielles de la gestion locale des ressources (ARNOLD, STEWART, 1991) ; - et surtout de réaffirmer de façon solennelle la légitimité refondée de cette gestion locale.

\section{Valoriser les ressources naturelles et financer le développement communal}

Dans un certain nombre de pays africains, en particulier anglophones, les réformes réglementaires ont eu souvent une orientation très marquée vers la conservation (ALDEN WILY, 2000) plutôt que vers la valorisation locale des ressources forestières. Cela marque une différence fondamentale avec les réformes engagées au Niger et au Mali qui ont largement été fondées sur la reconnaissance de l'importance économique des filières bois et en particulier bois énergie. De ce fait, ces réformes ont cherché d'emblée à ouvrir aux populations (et à améliorer) l'accès à des activités économiques et à des marchés rémunérateurs ${ }^{10}$. Cette meilleure valorisation des gestionnaires et producteurs primaires contribue à la mise en place d'une gestion locale viable à long terme, adaptée, des diverses ressources exploitées. L'idée de base, différente de celle de la simple préservation, étant que la durabilité de la gestion pourrait être une conséquence plus ou moins naturelle de la valorisation locale des ressources forestières et de la place irremplaçable que prendraient les produits forestiers dans le revenu des ménages ruraux. C'est sur la base de cette deuxième caractéristique, dont l'équivalent ne se retrouve pratiquement pas dans les pays africains anglophones, que la nécessité d'une gestion publique indirecte et fondée sur des instruments économiques des filières des produits forestiers s'est imposée au Niger et en apparence seulement au Mali.

\footnotetext{
8 Inventaire forestier réalisé avec l'appui du Cirad dans les années 1980.

9 Par exemple : feuilles de doum et gomme arabique au Niger.

${ }^{10}$ C'était un des objectifs de la réforme de la fiscalité forestière introduite au Niger par le projet Énergie II - Énergie domestique à partir de 1989.
} 
Trois caractéristiques essentielles différencient le transfert de gestion de ce qu'il est convenu d'appeler la participation :

- la reconnaissance de droits exclusifs locaux aux populations rurales sur les ressources forestières dans les limites du terroir ;

- une réelle autonomie de gestion de ces ressources forestières à leur profit dans le cadre de règles définissant les modalités de gestion et d'aménagement des forêts transférées, et souvent fixant aussi des règles pour l'utilisation des revenus locaux des activités forestières ;

- une fixation définie contractuellement comme permanente et intangible des limites des espaces forestiers transférés.

C'est le monopole local de gestion et de commercialisation mis en place par l'institution des marchés ruraux qui valorise cette innovation institutionnelle aux yeux des populations, plus que les dispositions de la fiscalité différentielle, même si ces dernières contribuent au Niger à ce bilan rural positif (Bertrand, MontAgne, 2006).

Au Niger, la création des structures locales de gestion a fait des populations locales les principaux acteurs de la gestion, de l'exploitation et de la sauvegarde des ressources forestières. Par des moyens variés et souvent propres à elles mêmes, elles ont été amenées à jouer le rôle de police forestière. Leurs espaces sylvopastoraux sont reconnus comme faisant partie de biens à conserver. Près des trois quarts des populations riveraines pensent que les marchés contribuent à conserver les ressources forestières et à protéger l'environnement.

Le monopole local de gestion et de commercialisation semble moins strict au Mali qu'au Niger, mais la légitimité locale retrouvée pour la gestion des espaces et des ressources forestières constitue probablement un des points importants si on doit tirer un bilan provisoire des marchés ruraux au Mali. On sait de façon assez argumentée que les

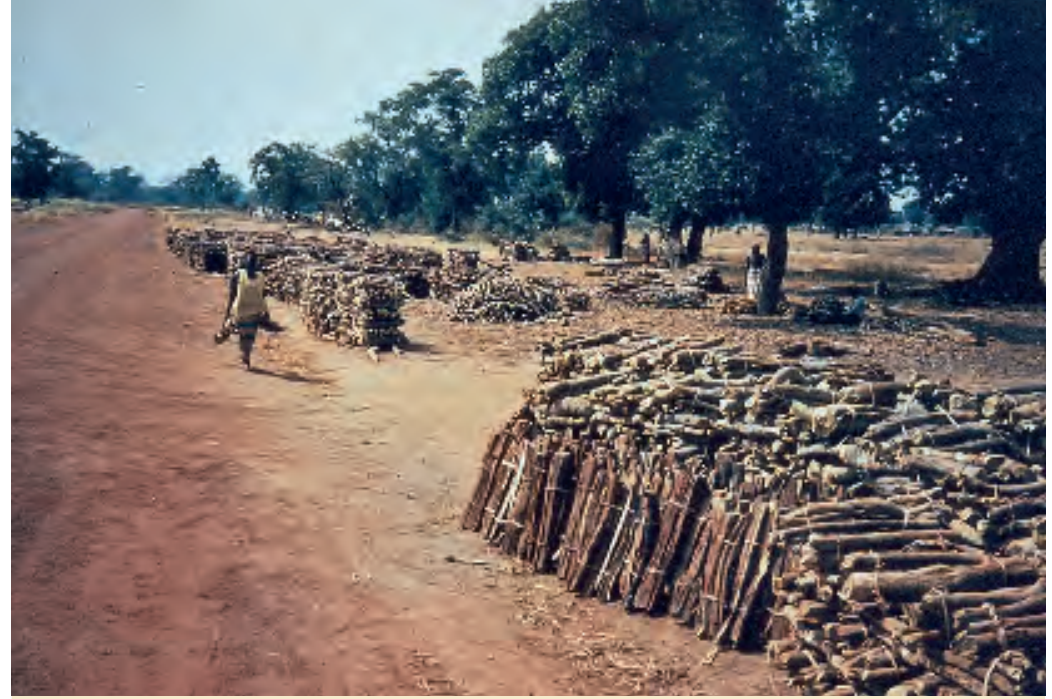

Un marché informel de bois sur la route Koulikoro-Banamba, au Mali, en 1985. Photo A. Bertrand.

populations rurales les plus pauvres ont pu tirer bénéfice de l'opportunité qui leur était faite d'exercer quasiment sans contrainte le bûcheronnage. Toutefois, on ne sait pas combien de temps cette opportunité économique est ouverte localement, compte tenu de la vitesse avec laquelle les essences les plus prisées sont exploitées et de la vitesse aussi avec laquelle l'activité bûcheronnage a été réinvestie par les « nantis » du village après un temps d'hésitation. C'est sur le plan social et territorial, en fait, que l'acquis est certainement le plus tranché : les villageois ont trouvé dans l'exploitation du bois une manière de réacquérir de la légitimité sur leurs territoires et leurs ressources.

Ainsi, au Mali, un certain nombre de nouveaux problèmes se posent dans le contexte de la décentralisation, et une question se pose quant à l'instrumentalisation, dans certains cas, de l'exploitation du bois par les autorités coutumières pour tenter de s'opposer aux nouvelles autorités communales et réasseoir leur légitimité mise à mal dans les premiers temps des marchés ruraux (GAUTIER et al., 2006).
Au Niger, à la suite de l'élaboration des schémas directeurs, du processus de développement des marchés ruraux, de la promulgation et mise en application des nouvelles règles fiscales qui réorganisent les conditions spatiales, techniques et économiques de l'approvisionnement en bois des villes, il s'avère que les flux financiers générés par l'exploitation issue des marchés ruraux sont très importants. Ils entraînent des changements considérables des comportements au niveau collectif des villages où des marchés ruraux ont été créés, mais aussi au niveau individuel des exploitations agricoles. Les marchés ruraux sont, pour les populations concernées, un moyen d'obtenir les financements nécessaires au renforcement des capacités collectives ou individuelles de développement. Si les sommes collectées sont insuffisantes pour mener une opération particulièrement coûteuse au vu des fonds disponibles, les différentes caisses villageoises assureront l'autofinancement nécessaire à l'obtention des crédits extérieurs. Ces chiffres sont encore modestes par rapport à l'importance du chiffre 
d'affaires annuel de la filière incontrôlée (de l'ordre de quatre cents millions de francs $\mathrm{Cfa}$ ) mais en représentent tout de même $15 \%$ et confirment donc l'impact global de la Sed. Une part importante des recettes fiscales est utilisée pour financer des investissements à usages collectifs (réparation de puits et forages, campagnes de vaccination, etc.) et appuyer des actions de développement rural (pépinières, confection de pare-feux, financement de stocks de sécurité alimentaire, etc.). L'importance des revenus financiers des marchés ruraux ainsi que l'orientation actuelle des investissements réalisés par les villages peuvent être la base d'actions de développement local, que cela soit sur des bases collectives au travers des différentes caisses ou individuelles au niveau des exploitations agricoles grâce aux revenus des bûcherons. Ainsi, les marchés ruraux sont, pour les villages où il est possible d'en créer, la "porte d'entrée » aux actions de développement local de type gestion des terroirs ou gestion des ressources naturelles (ISSAKA et al., 2006). Une étude (BAYARD-GAMATIÉ, MADON, 2003) montre, pour le Niger, que le revenu individuel de chaque bûcheron serait de l'ordre de cent mille francs Cfa par mois (ce qui représenterait de l'ordre d'un million de francs Cfa par an pour un bûcheron très impliqué). Cela souligne l'importance de ces revenus dans des budgets ménagers qui sont plus proches en moyenne de cent mille francs Cfa par an! Les conditions de vie des populations se sont donc considérablement améliorées; signe le plus manifeste: la diminution très importante de l'exode rural. Ces revenus sont redistribués dans l'exploitation agricole d'appartenance et permettent à ces exploitations d'assurer des besoins autres qu'alimentaires et de simple reproduction (scolarisation des enfants, embouche, baptêmes, mariages, intrants agricoles et charrettes, santé, habits, voyages, etc.).

\section{Domanialité} et fiscalité locale : quelle sécurisation foncière des communs?

Au Niger, les impacts socio-économiques sont donc significatifs sans pour autant que les ressources forestières soient détruites, notamment dans la mesure où cette exploitation villageoise n'a fait que se substituer à une exploitation qui, de toute façon, aurait eu lieu au profit des exploitants traditionnels. Nous avons aussi signalé que cette exploitation villageoise n'assure toujours, après près de quinze ans d'efforts, que $15 \%$ de la consommation d'une ville comme Niamey. La diminution des surfaces forestières reste plus une affaire liée aux défrichements agricoles qu'à l'exploitation ellemême. Par la délimitation des espaces forestiers réservés à l'exploitation ligneuse, les marchés ruraux jouent bien un double rôle : économique en améliorant les conditions de vie des familles des bûcherons, mais aussi social par la sécurisation foncière de ces espaces et leur soustraction à des volontés extérieures d'acquisition de domaines agricoles ${ }^{11}$.

Transférer la gestion des ressources renouvelables aux communautés rurales ne règle qu'une partie des problèmes fonciers : ceux qui concernent les espaces naturels. La décentralisation ouvre la possibilité de mutations plus ou moins profondes et plus ou moins rapides, mais de toute manière inéluctables, de la domanialité :

- au Niger, en 1992, dans un contexte de pouvoir centralisé et d'exception, le choix du transfert de gestion des formations naturelles aux communautés rurales s'est orienté vers la perspective de concessions rurales devant à terme déboucher sur une cession définitive matérialisée par l'immatriculation; depuis la loi 2004 040 du 8 juin 2004 fixant le Régime forestier, la possibilité d'un transfert de la domanialité sur les ressources forestières de l'État vers les collectivités territoriales décentralisées et en particulier vers les communes est ouverte au Niger ;

- au Mali, en 1998, dans un contexte de soudaine libéralisation politique, de démocratisation et de décentralisation, le choix a été clairement fait du transfert de la propriété du sol du domaine privé de l'État au domaine privé des collectivités territoriales décentralisées et en particulier des communes.

Au Mali, à la suite des bouleversements politiques consécutifs à la chute du régime de Moussa Traoré et sous la pression des populations rurales, le choix d'une décentralisation rapide a été fait. La création des communes s'est concrétisée à travers un certain nombre de textes réglementaires. En particulier, une loi a transféré du domaine privé de l'État au domaine privé des communes la propriété des ressources forestières. Mais que vaut une loi sans toute une chaîne de décrets et de textes d'application ? Après le reflux des exigences des populations rurales, le blocage de la situation malienne semble aujourd'hui durablement patent. Actuellement, les communes rurales du Mali ne peuvent pas effectivement bénéficier, de quelque manière que ce soit, des recettes de la fiscalité forestière. Tout se passe au Mali comme si l'administration forestière avait réussi à bloquer le transfert de la gestion des ressources naturelles aux populations rurales, non pas sur le plan des principes et de la loi, mais dans les modalités d'application.

11 Qui n'ont fonctionné que très imparfaitement en raison du faible taux de recouvrement sur l'exploitation incontrôlée. 


\section{Conclusion}

On voit que les deux principaux défis qui sont posés aujourd'hui au Niger et au Mali pour atteindre une gestion durable de leurs écosys tèmes forestiers concernent :

- la dévolution d'une part significative de la fiscalité forestière aux communautés villageoises et aux communes ; - l'organisation d'un système de contrôle forestier rénové et décentralisé associant les communautés de base, les communes et l'administration.

Ces deux défis se déclinent évidemment de façon différente au Niger et au Mali :

- au Niger, il s'agit, d'une part, de développer l'emprise spatiale globale des aménagements des formations forestières par le développement de nouveaux marchés ruraux pour généraliser l'exploitation contrôlée ${ }^{12}$ et, d'autre part, de passer d'un système inefficace de contrôle administratif de l'exploitation dans les zones incontrôlées à un système de contrôle efficace associant à la fois l'administration forestière, les communes et les communautés villageoises ;

- au Mali, il s'agit de tirer toutes les conséquences des choix faits en 1993 et 1996-97 pour remettre en chantier la réforme de la réglementation, de la fiscalité et du contrôle forestier afin de donner aux com. munes et aux communautés un rôle réel et des revenus locaux qui enclenchent un cercle vertueux en faveur de l'aménagement forestier durable.
12 Dont les sous-produits sont la commercialisation des produits ligneux qui assurent le financement de leur viabilisation. C'était déjà l'objectif du Pafn (Projet d'aménagement des forêts naturelles du Niger), qui a réalisé la mise sous aménagement durable de plus de 600000 ha de forêts au Niger et qui avait proposé un nouveau projet pour étendre encore ses actions.
En 2008, la hausse heureusement temporaire des prix des produits pétroliers a permis aux pays sahéliens de tirer un certain nombre de leçons que la baisse des prix récente et temporaire ne doit pas faire oublier. Les villes sahéliennes comme Niamey et Bamako sont condamnées à rester durablement consommatrices de bois énergie pour l'approvisionnement en énergie domestique des populations urbaines. L'économie du Niger ou du Mali ne supporterait pas la charge sur la balance des paiements qui résulterait d'un passage généralisé du bois énergie à des combustibles pétroliers (gaz butane, par exemple), même en période de bas coûts des produits pétroliers.

C'est un défi majeur à deux titres : - la lutte contre la désertification : une population urbaine croissante et des ressources forestières limitées et menacées par les évolutions climatiques et par l'aggravation de la désertification qui en résulte et en résultera au Niger, mais aussi au Mali et ailleurs au Sahel ;

- assurer un approvisionnement en énergie domestique écologiquement durable et socialement stable et économe ; il devient nécessaire de généraliser à l'échelle de la totalité du bassin d'approvisionnement des villes (comme par exemple la ville de Niamey) la mise en place effective d'une gestion soutenue, durable et robuste des forêts.

Les enjeux sont la vie quotidienne des populations urbaines, l'équilibre social et le maintien d'un équilibre environnemental fragile et menacé. 


\section{Références bibliographiques}

ALDEN WILY L., 2000. La législation forestière en Afrique orientale et australe : vers un avenir forestier à orientation communautaire ? Unasylva, 203, vol. 51 : 19-26.

ANONYME, 1996. Rapport de fin de mission de l'assistance technique au projet Pafn. Niamey, Niger, Cirad/Pafn.

ANONYME, 2005. Rapport d'évaluation des marchés ruraux de bois mis en place par la SED phase 1. Bamako, Mali, Beagges.

ARNOLD J. E. M., STEWART W. C., 1991. Common Property Resource Management in India. Oxford, Royaume-Uni, Oxford Forestry Institute, University of Oxford, Tropical Forestry Papers $n^{\circ} 24$.

BAYARD-GAMATIÉ M., MADON G., 2003. Le bilan dix ans après la création des marchés ruraux au Niger : une responsabilisation réelle des populations rurales pour la gestion et le contrôle des ressources ligneuses de leurs terroirs. Niamey, Niger, Pafn. BERTRAND A., MONTAGNE P., 2006. Quel bilan tirer de la fiscalité forestière différentielle sur le bois énergie au Niger ? In : Bertrand A., Montagne P., Karsenty A. (éd.). L'État et la gestion locale durable des forêts en Afrique francophone et à Madagascar. Paris, France, L'Harmattan.
BERTRAND A., MONTAGNE P., BESSE F., SERRE-DUHEM C., RAHARIMANIRAKE L., 2006. Nouvelles politiques forestières et gestion économique publique des filières des produits forestiers non ligneux. In : Bertrand A., Montagne P., Karsenty A. (éd.). Forêts tropicales et mondialisation : les mutations des politiques forestières en Afrique francophone et à Madagascar. Paris, France, L'Harmattan.

BERTRAND A., MONTAGNE P., 2008. Domanialité, fiscalité et contrôle : la gouvernance locale contractuelle des ressources renouvelables dans un contexte de décentralisation (Niger, Mali et Madagascar). Mondes en développement, 36 (141) : 11-28.

GAUTIER D., HAUTDIDIER B., KEITA S., MBODJ F., 2006. Coupe de bois et revendications sociales au Mali. In : Colloque international "Les frontières de la question foncière. Enchâssement social des droits et politiques publiques ", Montpellier, 17-19 mai 2006.

HARDIN G., 1968. The tragedy of the commons. Science, 162 : 1243-1248.
ISSAKA H., HAMADOU M., MAMAN R., 2006. La gestion du marché rural de bois énergie et le développement local de Kankani au Niger. In : Bertrand A., Montagne P., Karsenty A. (éd.). L'État et la gestion locale durable des forêts en Afrique francophone et à Madagascar. Paris, France, L'Harmattan.

KANOUTÉ A., 2005. Mesure de l'impact socio-économique des structures rurales de gestion de bois créées dans les bassins d'approvisionnement en bois de Bougouni et Sikasso. Bamako, Mali, Sps Gie, Beagges.

MADON G., MATLY M., 1986. Conservation et substitution de l'énergie à usage domestique. Projet Unso/Ner/85/X02. Sema-énergie.

MONTAGNE P., RAZANAMAHARO Z., COOKE A., 2007. Le transfert de gestion à Madagascar, dix ans d'efforts : Tanteza. Montpellier, France, Cirad.

ROY S. B., 1995. Enabling environment for joint forest management. New Delhi, Inde, Inter-India Publications, Forest Studies Series F 004. 